\title{
User manual for IDENT, a parametric and nonparametric linear systems identification package
}

\author{
Susan A.S. Werness and David J. Anderson \\ Kresge Hearing Research Institute, University of Michigan, Ann Arbor, MI 48109, USA
}

\section{INTRODUCTION}

This document is intended to provide a first time user of IDENT with tutorials on the usage of the program. The user should be familiar with the theoretical aspects of linear system identification. The Box and Jenkins (1976) Time Series Analysis is probably the best first reference. Following are explanations of each command (generated by IDENT), and examples of each command.

IDENT uses 2 types of input files. One type contains the input and output data to be analyzed. The other type contains commands with which the program may be run automatically. It has been the experience of this laboratory that the automatic mode of running IDENT is the only one used. Usually, one wants to estimate and evaulate several models before choosing a model structure. On an LSI-11, the time involved in such an effort is considerable, so that modeling was often done in an overnight run of IDENT.

COMAND NUMBER 1

IDENTIFICATION OF THE OUTPUT ARRAY

FUNCTION OE THE COMMAND:

This command results in a univariate analysis of some
fraction of the possibly detrended or differenced output data array.

See the comments about command 8 for details concerning the establishment of the data case range, for setting up the differencing factor, and for control over whether the data are detrended.
UNIVARIATE ANALYSIS:

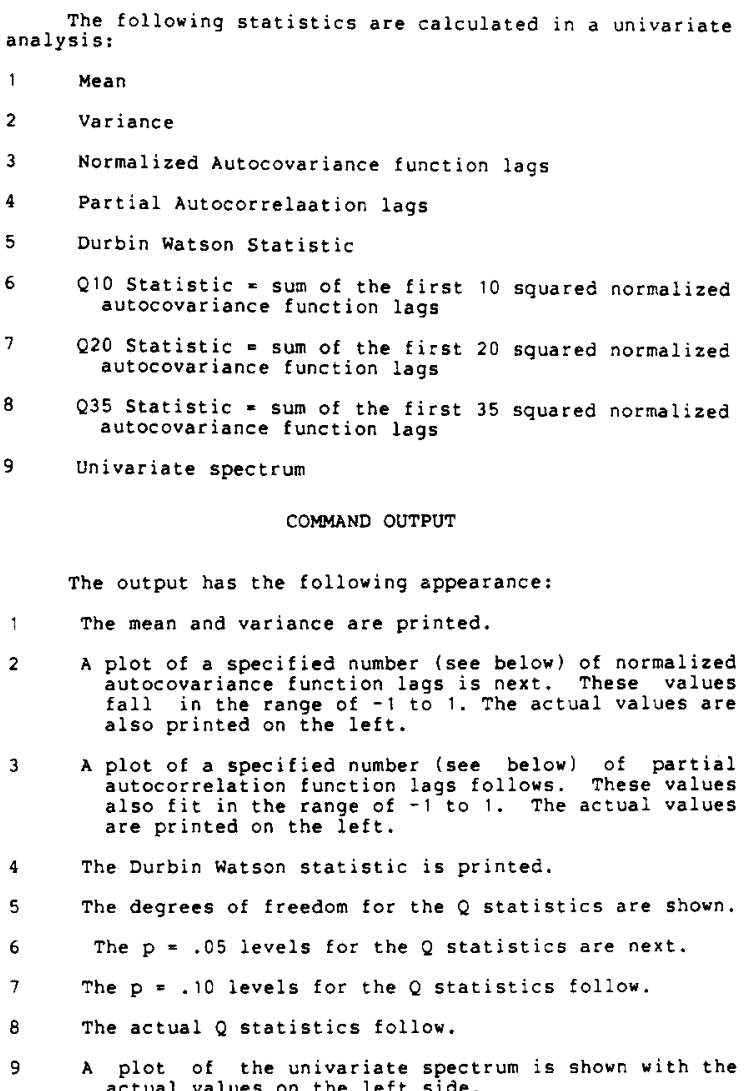

COMMAND OUTPUT

The output has the following appearance:

The mean and variance are printed.

A plot of a specified number (see below) of normalized autocovariance function lags is next. These values fall in the range of -1 to 1 . The actual values are also printed on the left.

3 A plot of a specified number (see below) of partial autocorrelation function lags follows. These values also $f$ it in the range of -1 to 1 . The actual values are printed on the left.

The Durbin Watson statistic is printed.

The degrees of freedom for the $Q$ statistics are shown.

The $p=.05$ levels for the $Q$ statistics are next.

The $p=.10$ levels for the $Q$ statistics follow.

The actual $Q$ statistics follow.

A plot of the univariate spectrum is shown with the USER CONTROL OVER THE COMMAND

The user is not required to enter any input.

Certain details of the command are controlled by operation parameters. Any operation parameters may be changed by the command 9 . 
The NA operation parameter controls the number of normalized autocovariance lags plotted. It has a default
value of 10 .

The NP operation parameter controls the number of partial autocorrelation lags plotted. It has a default value

The KSAMP operation parameter is the resampling factor. The user can control the upper frequency of the spectral plots with this parameter. It can take on the values $1,2,4$, or 8 corresponding to the entire, half. fourth, or eighth of the Nyquist frequency $(1 / 2$ the sampling rate. It has a default value of 8 .

size of the window operation parameter, LAG, controls the spectrum. It lag window used in the smoothing of the a window length of $1 / 2$ or $1 / 4$ the array size. A smoother spectral estimate will result with a value of 2 . It has a defaut value of 1 .

Examrle of command 1

*********IIENTIFICATIDN OF OUTFUT ARRAY***********

MEAN $=\quad-0.00000$ VAFIANCE $=4.36856$

-AUTOCOFFELATIONS-

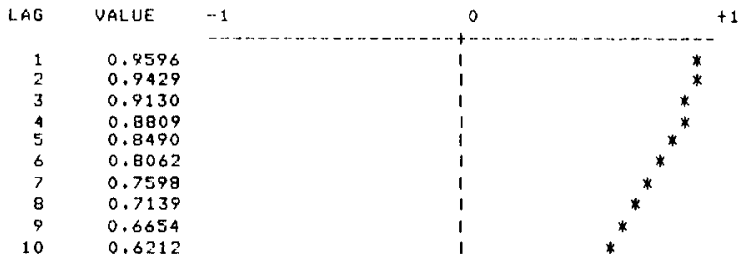

-FARTIAL AUTOCORFELATIONS-

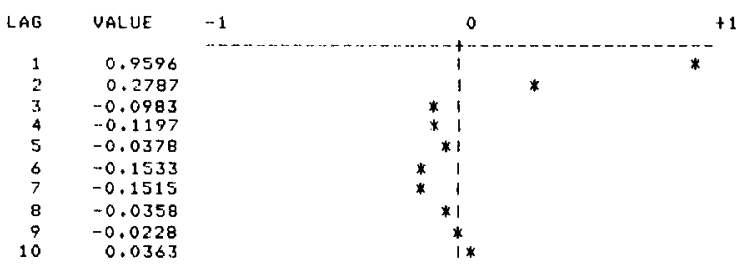

DURHIN-WATSON STATISTIC $=\quad 0.07492$

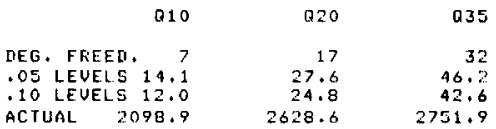

SFECTRUM

\begin{tabular}{|c|c|c|}
\hline & IIENS ITY & $-t-\cdots$ \\
\hline$\stackrel{0}{1}$ & $\begin{array}{l}148.0702 \\
140.1490\end{array}$ & 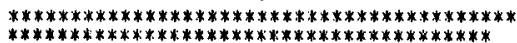 \\
\hline 2 & 118.9074 & $* * * * * * * * * * * * * * * * * * * * * * * * * * * * * * * * * * *$ \\
\hline 3 & 92.2721 & 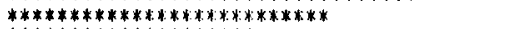 \\
\hline 4 & 70.7658 & $* * * * * * * * * * * * * * * * * * * *$ \\
\hline 5 & 60.1605 & 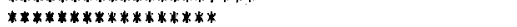 \\
\hline 6 & 58.2954 & $* * * * * * * * * * * * * * * *$ \\
\hline 7 & 60.7174 & ******************1 \\
\hline 8 & 65.2456 & *********************1 \\
\hline 9 & 68.5665 & $* * * * * * * * * * * * * * * * * * *$ \\
\hline 10 & 63.9055 & $* * * * * * * * * * * * * * k * * *$ \\
\hline 11 & 48.1959 & $* * * * * * * * * * * * *$ \\
\hline 12 & $28.196 ?$ & **********1. \\
\hline
\end{tabular}

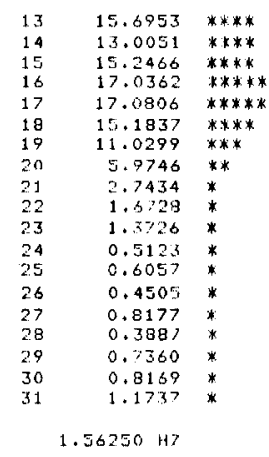

COMMANI:

COMMAND NUMBER

PARAMETER ESTIMATION

FUNCTION OF THE COMAND:

This command results in the estimation of parameters of a specified model structure based on some fraction of the possibly detrended or differenced output and input data.

See the comments about command 8 for details concerning the establishment of the data case range, for setting up the differencing factor, and for control over whether the data are detrended.

In order to be able later to do a complete evaluation of a transfer function model, a 6 command should be done prior to a parameter estimation so that a prewhitened input series is available. (see comments under command 6 and command 3.) A prewhitened input cannot be obtained after a parameter estimation without destroying the parameter estimates.

COMMAND OUTPUT

The output has the following appearance:

The deadtime factor ( the number of data points the output is assumed to be lagging the input) is printed.

2 The model orders are printed with abbreviated labels:

a) $T F N=t r a n s f e r$ function numerator

b) $T F D=$ transfer function denominator = noise

c) NN noise model numerator

d) $\mathrm{ND}=$ noise model denominator

3 For each iteration, the parameter estimates are witten in the order: TFN, TFD, NN, ND.

4 At the last iteration, standard deviations of the parameter estimates and the stochastic approximation estimate of the residual variance are written.

USER CONTROL OVER THE COMMAND

The model orders (transfer function poles, transfer function zeros, noise model poles, noise model zeros) and deadtime factor must be supplied from a command file or in response to the queries:

ENTER ORDERS: TFP, TFZ, NP, NZ

ENTER DEADTIME FACTOR:

Positive integers should be entered. Any of these orders may be zero to result in the estimation of a subset of a transfer function plus noise model. If TFP is 0 and TFZ is -1 , then the parameters of a univariate ARIMA model are estimated. 


\section{**********MOIEL FAFAHETEF ESTIMATION***********}

ENTER ORIEKS: TFF, IFZ, NF, NZ $1,0,1,2$ ENTEK IELAY FACTOR 4

THE DEALITIME: FACTOR

NUMEER OF FARAMETIRS:TFH: 1 TFI: 0 NM: 2 NI:

ITEKATION

PARTAMETERS

$\begin{array}{rr}0 & -0.11414 \\ 1 & -0.03937 \\ 2 & -0.02809 \\ 3 & -0.00855 \\ 4 & 0.00780 \\ 5 & 0.02352 \\ 6 & 0.04611 \\ 7 & 0.06599 \\ 8 & 0.07748 \\ 9 & 0.08316 \\ 10 & 0.08620\end{array}$

$\begin{array}{rr}-0.23169 & -0.06846 \\ -0.46698 & 0.19644 \\ -0.19657 & -0.09644 \\ 0.03256 & -0.33474 \\ 0.25573 & -0.56264 \\ 0.52625 & -0.83591 \\ 0.73623 & -1.05175 \\ 0.85461 & -1.17670 \\ 0.89334 & -1.22098 \\ 0.90675 & -1.23752 \\ 0.91248 & -1.24473\end{array}$

0.09905

0.04431

0.182440 .9773

0.242320 .97842

$0.31313 \quad 0.97737$

$0.36893-0.97442$

$0.39213 \quad 0.96910$

$0.39637 \quad 0.96329$

$0.39711 \quad 0.95752$

$0.39723 \quad 0.95200$

ESTIMATEO STANIAREI IIEUIATIONS:

$\begin{array}{llllll}\text { STD DEV. } & 0.01154 & 0.01994 & 0.02549 & 0.01893 & 0.00737\end{array}$ THE RECURSIVELY ESTIMATEL RESIIUAL. VARIANCE:= 0.24715

COMMANII:

\section{COMMAND NUMBER}

\section{MODEL EVALUATION}

FUNCTION OF THE COMAAND:

In the evaluation of a general transfer function plus noise model, the following computations are performed:

Calculation of residual series

Univariate analysis of the residuals

A specified number of cross correlation lags between the residual and prewhitened input are calculated.

S11 statistic = the first 11 squared normalized cross correlations summed

S21 statistic = the sum of the first 21 squared normalized cross correlations $\$ 36$ statistic $=$ the sum of the first 36 squared
normalized cross correlations

The parametric transfer function

The signal variance = variance of the parametric transfer function

The noise variance = variance of the parametric noise model

The signal variance to noise variance ratio

Poles and zeros of the parametric transfer function and noise model

If the model being evaluated is a univariate one Inoise model onlyl, then steps $3,4,5,6,7,8,9$, and 10 above are omitted.

The following statistics are calculated in a univariate analysis:

Mean

Variance

Normalized Autocovariance function lags

Partial Autocorrelaation lags

Durbin Watson Statistic
Q10 Statistic $=$ sum of the first 10 squared normalized autocovariance function lags

7 Q20 Statistic $=$ sum of the first 20 squared normalized autocovariance function lags

Q35 Statistic $=$ sum of the first 35 squared normalized autocovariance function lags

Univariate spectrum

COMMAND OUTPUT

For the univariate residual analysis:

The output has the following appearance:

The mean and variance are printed.

2 A plot of a specified number (see below) of normalized autocovariance function lags is next. These values fall in the range of -1 to 1 . The actual values are also printed on the left.

3 A plot of a specified number (see below) of partial autocorrelation function lags follows. These values also $f$ it in the range of -1 to 1 . The actual values are printed on the left.

4 The Durbin watson statistic is printed.

5 The degrees of freedom for the $Q$ statistics are shown.

6 The $p=.05$ levels for the $Q$ statistics are next.

7 The $p=.10$ levels for the $Q$ statistics follow.

8 The actual $Q$ statistics follow.

9 A plot of the univariate spectrum is shown with the actual values on the left side.

For the residual analysis, the spectrum is not plotted.

The output from the bivariate analysis between the residuals and the prewhitened input has the following appearance:

1 The mean and variance of the residuals are printed.

2 The mean and variance of the prewhitened input are next.

3 The cross correlation coefficient (normalized cross correlation at lag 0 ) is printed.

4 A plot of a specified number (see below) of normalized cross correlation lags is next. These values fall in the range of -1 to 1 . The actual values are printed on the left.

5 The degrees of $f$ reedom for the $S$ statistics are shown.

6 The $p=.05$ levels for the $s$ statistics are next.

7 The $p=.10$ levels for the $s$ statistics follow.

8 The actual s statistics (labeled $Q$ ) follow.

In the case of a transfer function model, the results of the evaluation of the parametric transfer function are typed:

The noise variance, signal variance, and signal to noise ratio, labeled $N, S, S N R$ are printed.

2 Sixteen values from $O \mathrm{~Hz}$ up to some fraction of the Nyquist frequency (see below) of the gain and phase of the parametric transfer function are plotted with the actual values printed on the left.

For all models, the values of the magnitudes and frequencies of the poles and zeros derived from the

USER CONTROL OVER THE COMMAND

The user is not required to enter any input for a model evaluation. 
Certain details of the command are controlled by operation parameters. Any operation parameters may be changed by the comand 9

The NA operation parameter controls the number of normalized autocovariance lags plotted. It has a default value of 10 .

The NA operation parameter controls the number of normalized cross correlation lags plotted. It has a default value of 10 .

The NP operation parameter controls the number of partial autocorrelation lags plotted. It has a default value of 10 .

The KSAMP operation parameter is the resampling factor. The user can control the upper frequency of the spectral plots with this parameter. It can take on the values $1,2,4$, or 8 corresponding to the entire, half fourth, or eighth of the Nyquist frequency (1/2 the sampling rate. It has a default value of 8 .

\section{Example of command 3}

\section{**********IIIAGNOSTICS************}

THE CURKENT CASE RANGE : 24508

-...-KESILUNL STOAISIICS -....-

MEAN $=0.00224$ VARIANCE.$\quad 0.21791$

-AUTOCORRELATIONS-

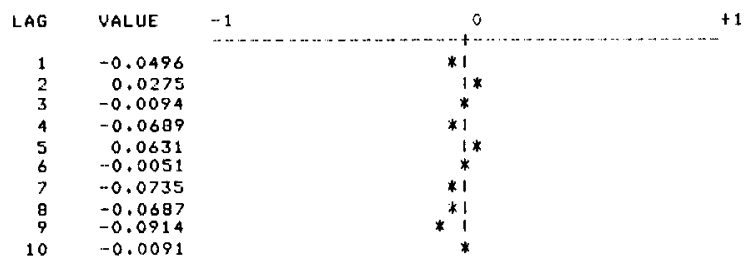

-FARTIAL AUTOCORKELATIONS.

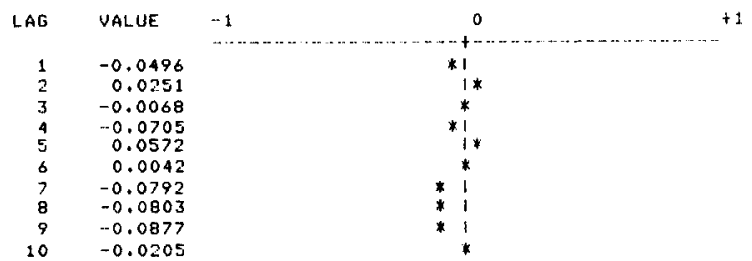

DUREIN-WATSON STATISTIC $=2.09915$

$\begin{array}{lrrr} & 010 & 020 & 035 \\ \text { DEG. FREEIT, } & 7 & 17 & 32 \\ .05 \text { LEVELS } & 14.1 & 27.6 & 46.2 \\ \text { 10 LEUELS } 12.0 & 24.8 & 42.6 \\ \text { ACTUAL } & 14.8 & 28.7 & 42.4 \\ & & & \end{array}$

$\begin{array}{lll}\text { MEAN }= & 0.00224 \text { VARIANCE }= & 0.31741 \\ \text { MEAN }= & 0.00046 \text { VARIANCE }= & 0.06410\end{array}$

THE CROSS- CORRELATION COEFFICIENT $\quad-0.047739$
-CROSS CORKELATIDNS.

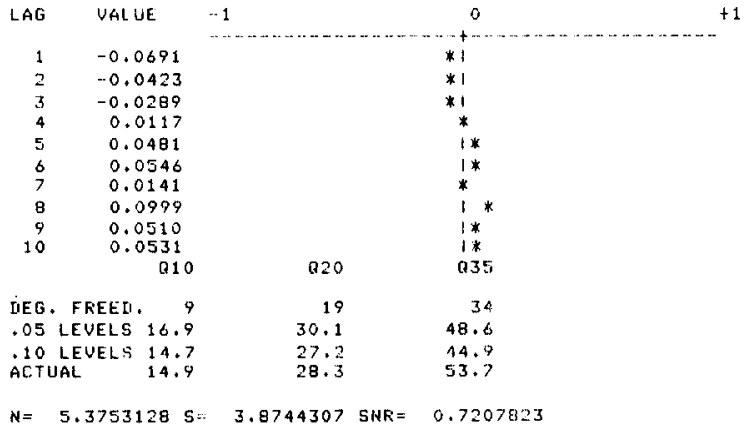

PARANETRIC TRANSFER FUNCTION SFECTRUM

ISAIN

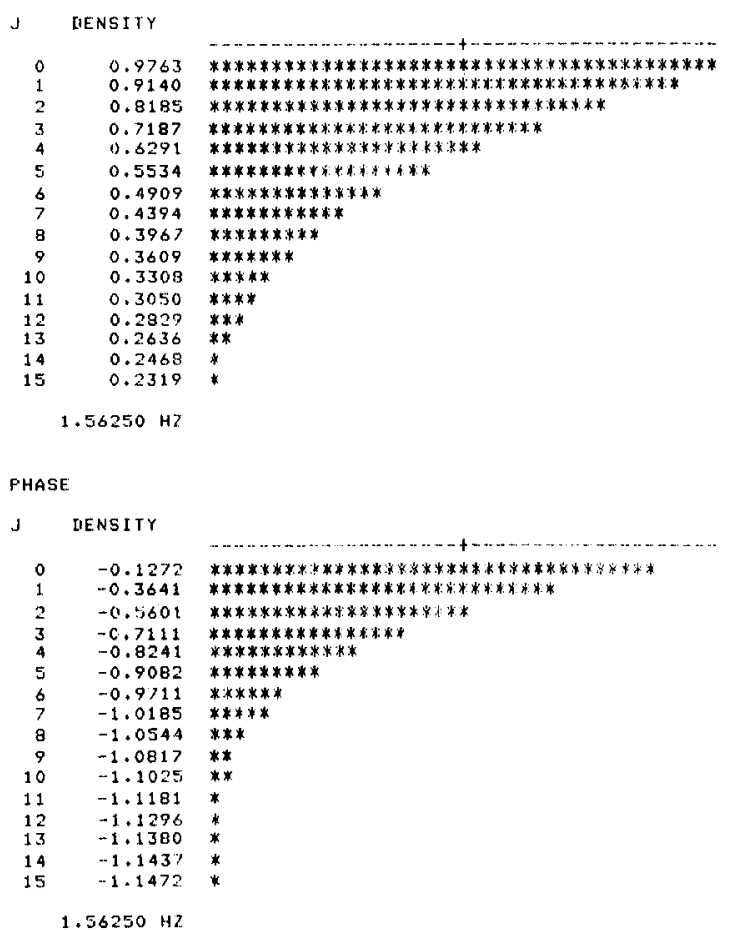

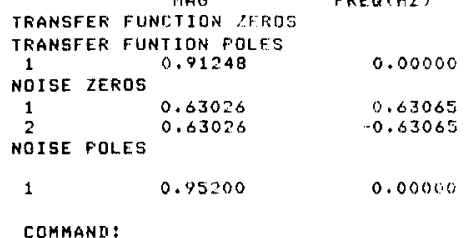

COMMAND: 
COMMAND MUNGER 4

IDENTIFICATION OF THE INPUT ARRAY

FUNCTION OF THE COMMAND:

This command results in a univariate analysis of some fraction of the possibly detrended or differenced output data array.

See the comments about command 8 for details concerning the establishment of the data case range, for setting up the differencing factor, and for control over whether the data are detrended.

UNIVARIATE ANALYSIS:

The following statistics are calculated in a univariate analysis:

mean

Variance

Normalized Autocovariance function lags

Partial Autocorrelaation lags

Durbin watson Statistic

Q10 Statistic = sum of the first 10 squared normalized autocovariance function lags

220 Statistic = sum of the first 20 squared normalized autocovariance function lags

Q35 Statistic = sum of the first 35 squared normalized autocovariance function lags

Univariate spectrum

COMMAND OUTPUT

The output has the following appearance:

The mean and variance are printed.

2 A plot of a specified number (see below) of normalized autocovariance function lags is next. These values fall in the range of -1 to 1 . The actual values are also printed on the left.

3 A plot of a specified number (see below) of partial autocorrelation function lags follows. These values also $f$ it in the range of -1 to 1 . The actual values are printed on the left.

4 The Durbin watson statistic is printed.

5 The degrees of freedom for the $Q$ statistics are shown.

6 The $p=.05$ levels for the $Q$ statistics are next.

The $p=.10$ levels for the $Q$ statistics follow.

The actual Q statistics follow.

9 A plot of the univariate spectrum is shown with the actual values on the left side.

USER CONTROL OVER THE COMMAND

The user is not reguired to enter any input.

Certain details of the command are controlled by operation parameters. Any operation parameters may be changed by the command 9 .

The Na operation parameter controls the number of normalized autocovariance lags plotted. It has a default value of 10 .

The NP operation parameter controls the number of partial autocorrelation lags plotted. It has a default value of 10 .

The KSAMP operation parameter is the resampling factor. The user can control the upper frequency of the spectral plots with this parameter. It can take on the values $1,2,4$, or $B$ corresponding to the entire, half, fourth, or eighth of the Nyquist frequency $(1 / 2$ the sampling rate. It has a default value of 8 .

The lagwindow operation parameter, LAG, controls the size of the lag window used in the smoothing of the spectrum. It may take on the values 1 or 2 , corresponding to a window length of $1 / 2$ or $1 / 4$ the array size. A smoother spectral estimate will result with a value of 2 , It has a default value of 1 .

Evamp te of cominas 4

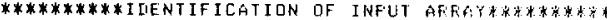

MEAN = $\quad 0.00000$ VAF:IANCE: $\quad 3.71093$

-AUTOCORFELATIONS-

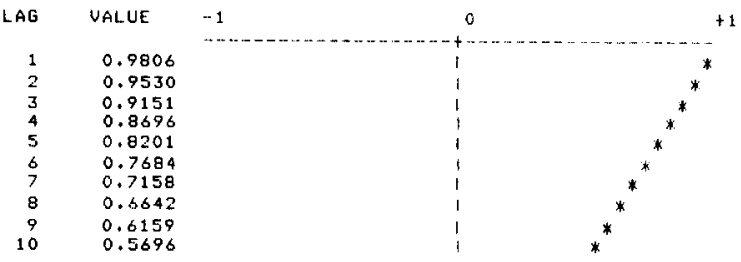

-FARIIAL AUTOCOFFELATIONS.

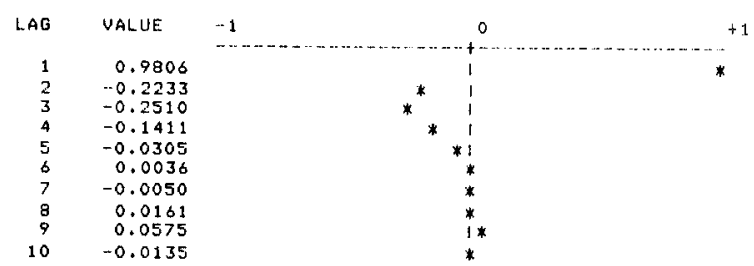

IURBIN-WAISON STATISTIC $=0.02354$

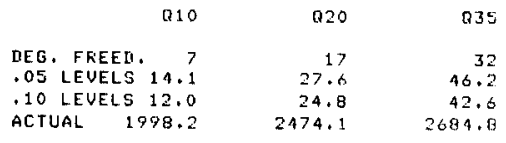

SFECTRUM

$J$ IENSITY

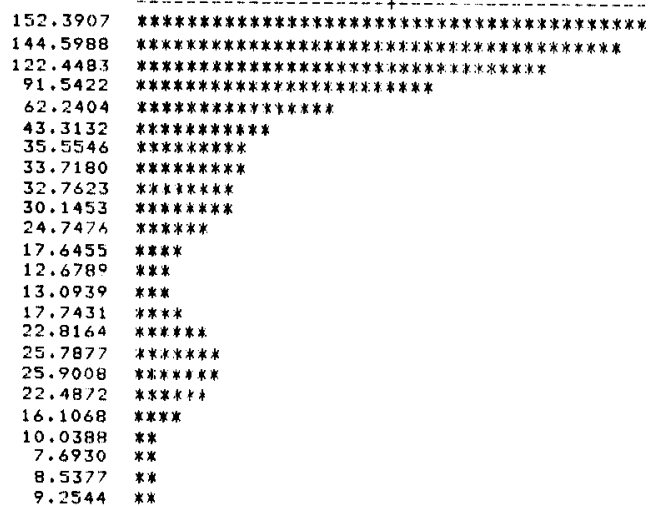




$\begin{array}{lrl}24 & 8.0104 & * * \\ 25 & 5.9195 & * \\ 26 & 4.2875 & * \\ 27 & 3.2764 & * \\ 28 & 2.5239 & * \\ 29 & 2.1555 & * \\ 30 & 1.8422 & * \\ 31 & 1.5495 & * \\ & 1.56250 \mathrm{H3} & \end{array}$

COMMAND:

COMGAMD NUKBER 5

\section{IDENTIFICATION OF THE NOISE ARRAY}

FUNCTION OF THE COMMAND:

This command results in the estimation of a correlated noise series. A truncated version of the impulse response obtained nonparametrically from command 7 is used to compute estimates of the transfer function output, which are subtracted from the actual output to get an estimate of the noise component. A univariate analysis is done on the estimated noise series.

UNIVARIATE ANALYGIS:

The following statistics are calculated in a univariate analysis:

Mean

Variance

Normalized Autocovariance function lags

Partial Autocorrelation lags

Durbin Watson Statistic

Q10 Statistic = sum of the first 10 squared normalized autocovariance function lags

Q20 Statistic = sum of the first 20 squared normalized autocovariance function lags

Q35 Statistic = sum of the first 35 squared normalized autocovariance function lags

Univariate spectrum

COMMAND OUTPUT

The output has the following appearance:

The mean and variance are printed.

A plot of a specified number (see below) of normalized autocovariance function lags is next. These values fall in the range of -1 to 1 . The actual values are also printed on the left.

3 A plot of a specified number (see below) of partial inection lags follows. These also fit in the range of -1 to 1 . The actual values are printed on the left.

4 The Durbin watson statistic is printed.

5 The degrees of freedom for the $Q$ statistics are shown.

6 The $p=.05$ levels for the $Q$ statistics are next.

7 The $p=.10$ levels for the $Q$ statisties follow.

8 The actual $Q$ statistics follow.

9 A plot of the univariate spectrum is shown with the actual values on the left side.

USER CONTROL OVER THE COMMAND

The user is not required to enter any input.
Certain details of the command are controlled by operation parameters. Any operation parameters may be changed by the command 9 .

The KSAMP operation parameter is the resampling factor. The upper frequency present in the nonparametricali derived impulse response is controlled by this parameter. I can take on the values $1,2,4$, or 8 corresponding to the entire, half, fourth, or eighth of the Nyquist frequency (1/2 the sampling rate. The input and output data are resampled according to KSAMP before estimation of the noise series. It has a default value of 8 .

The length of the estimated impulse response used to calculate the noise series is controlled by the operation parameter, IMP. It has a default value of 30 . It should be large enough so that significant values of the impulse response are not missed.

The NA operation parameter controls the number of normalized autocovariance lags plotted. It has a default value of 10 .

The NF operation parameter controls the number of partial autocorrelation lags plotted. It has a default value of 10 .

The lag window operation parameter, LAG, controls the size of the lag window used in the smoothing of the spectrum. It may take on the values 1 or 2 , corresponding to spectral estimate will result with a value of 2 . It has a defaut value of 1 .

Example of command 5

5

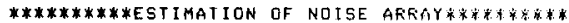

**********IIIENTIFICATION OF NOISE ARFAY***********

MEAN =

0.03532 VAKIANCE $-\quad 5.76799$

-AUTOCOKRELATIONS-

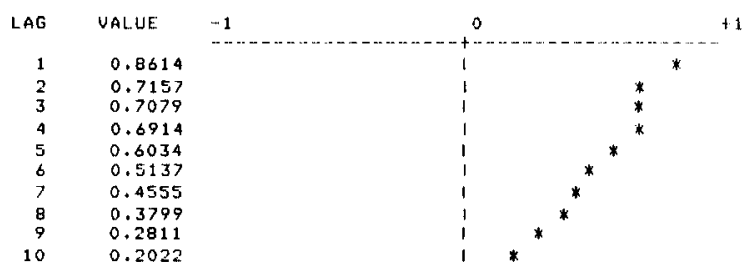

-FARIIAL AUTOCOFFELATIONS-

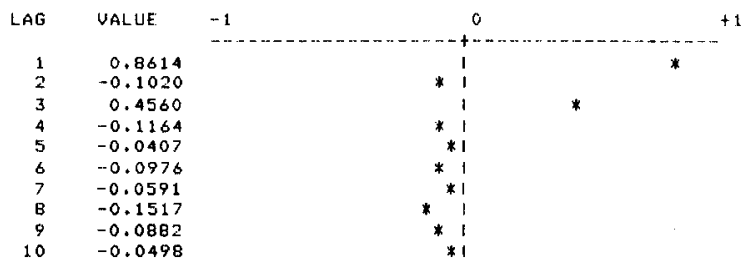

DUKBIN-WATSON STATISTIC $=$

$010 \quad 020 \quad 035$

DEG, FREED, 10

.05 LEVEIS 18.3

110 LEVELS 16.0

SPECTRUM

SPECTRUM 
COYOAND NUMBER 6

PREWHITEN THE INPUT

FUNCTION OF THE COMMAND:

This command results in the estimation of a specified number (see below) of autoregressive parameters with which to approximate the input series. The resulting model of the input is evaluated (as a univariate model). The residuals are set aside as the prewhitened input to be used in future detrended or differenced. Any fraction of the input array may be used.

See the comments about command 8 for details concerning the establishment of the data case range, for setting up the differencing factor and for control over whether the dat are detrended.

In the evaluation of a univariate model, the following computations are performed:

Calculation of the residual series

Univariate analysis of the residuals

Poles and zeros of the model

For the univariate residual analysis:

The following statistics are calculated in a univariate

\section{Mean}

Variance

Normalized Autocovariance function lags

Partial Autocorrelaation lags

Durbin watson Statistic

Q10 Statistic $=$ sum of the first 10 squared normalized autocovariance function lags

Q20 Statistic = sum of the first 20 squared normalized autocovariance function lags

Q35 Statistic = sum of the first 35 squared normalized a ucovariance function lags

Univariate spectrum

For the residual analysis, the spectrum is not plotted.

COMMAND OUTPUT

Since the autoregressive parameters of the input model are found using the same algorithm for the determination of the partial autocorrelation coefficents in a univariate analysis, the output from this command looks like 2 univariate analyses without spectra: 1) a univariate analysis of the input series followed by anivariate input she univariate analysis output is

The output has the following appearance:

The mean and variance are printed.

A plot of a specified number (see below) of normalized autocovariance function lags is next. These values fall in the range of -1 to 1 . The actual values are also printed on the left.

A plot of a specified number (see below) of partial autocorrelation function lags follows. These values also fit in the range of -1 to 1 . The actual values are printed on the left.

The Durbin Watson statistic is printed.

The degrees of freedom for the $Q$ statistics are shown.

The $p=.05$ levels for the $Q$ statistics are next.

The $p=.10$ levels for the $Q$ statistics follow.

The actual $Q$ statistics follow. 9 A plot of the univariate spectrum is shown with the
actual values on the left side.

USER CONTROL OVER THE COMMAND

The user is not required to enter any input.

Certain details of the command are controlied by operation parameters. Any operation parameters may be changed by the command 9 .

The NA operation parameter controls the number of normalized autocovariance lags plotted. It has a default value of 10 .

For the residual analysis:

The NP operation parameter controls the number of partial autocorrelation lags plotted. It has a default value of 10 .

For the input univariate analysis:

The NPW parameter controls the number of partial autocorrelation lags plotted. It has a default value of 17. NPW is the number of autoregressive parameters with which the input is approximated.

COMMAND OUTPUT

The command output consists of 3 plots with actual values printed on the left:

1 Sixteen values from $0 \mathrm{~Hz}$ up to some fraction of Nyquist freqency (see below) of transfer function
gain

2 Sixteen values from $0 \mathrm{~Hz}$ up to the same ffequency above of transfer function phase lags in radians

3 Fifty values of the impulse response

Example of command

*******ESTIMATION OF FREWHITENING PARAMETERS********

MEAN

$$
0.00012 \text { VARIANCE }=0.08078
$$

-AUTOCORFELATIONS -

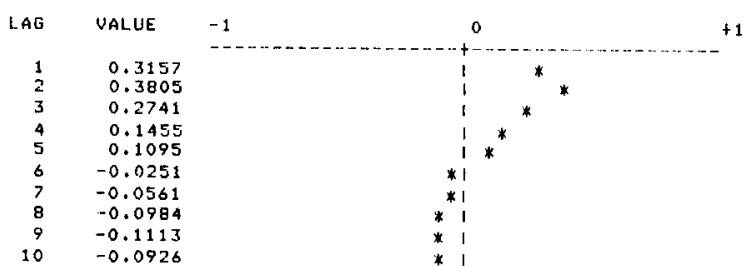

-FARTIAL. AUTOCORRELATIONS-

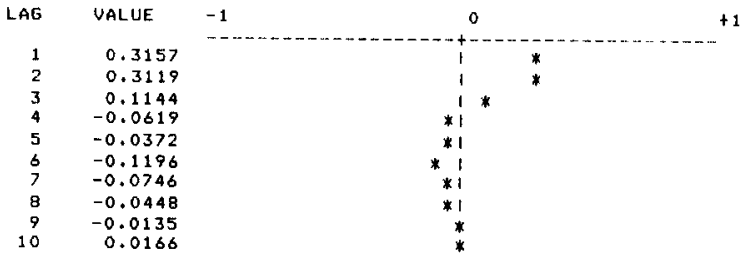




$\begin{array}{lr}11 & -0.0218 \\ 12 & -0.0293 \\ 13 & 0.0151 \\ 14 & -0.0136 \\ 15 & -0.0248 \\ 16 & 0.0170 \\ 17 & -0.0722\end{array}$

\begin{tabular}{|c|c|c|}
\hline DUREIN-WATSON ST & $\mathrm{I} C=$ & 1.36759 \\
\hline a10 & 020 & Q35 \\
\hline $\begin{array}{l}\text { IHEG. FREED, } 10 \\
.05 \text { LEVELS } 18.3 \\
10 \text { LEVELS } 16.0 \\
\text { ACTUAL } 197.8\end{array}$ & $\begin{array}{r}20 \\
31.4 \\
28.4 \\
221.9\end{array}$ & $\begin{array}{r}35 \\
49.8 \\
46.1 \\
248.0\end{array}$ \\
\hline
\end{tabular}

THE CUREENT CASE RANGE $=19 \$ 12$

-..--KESIDUAL STATISTICS......

MEAN $=0.00107$ VAFIANCE $=0.06390$

-AUTOCORGELATIONS-

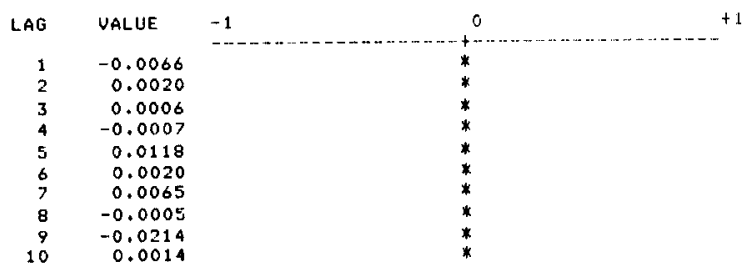

-FARTIAL AUTOCORRELATIONS-

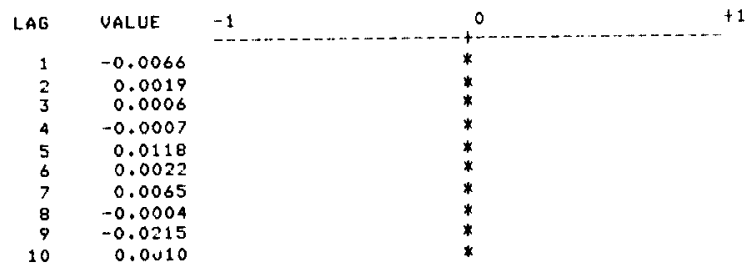

\begin{tabular}{lrrr} 
DUREIN-WATSON STAIISTIC & \multicolumn{1}{c}{2.01268} \\
& 010 & 020 & 0.35 \\
DEG. FREEI. & 0 & 3 & 18 \\
.05 LEVELS & 0.0 & 7.8 & 28.9 \\
.10 LEVELS & 0.0 & 6.2 & 26.0 \\
ACIUAL & 0.3 & 3.0 & 21.3
\end{tabular}

$$
\text { MAG FREQ(HZ) }
$$

\section{TRANSFER FUNCTION ZEROS} TRANSFER FUNTION POLES

NOISE ZEROS

NOISE POLES

$\begin{array}{rrr}1 & 0.88502 & \\ 2 & 0.88502 & -9.60281 \\ 3 & 0.87100 & 10.99081 \\ 4 & 0.87100 & -10.99081 \\ 5 & 0.86045 & 12.50001 \\ 6 & 0.86343 & 7.97997 \\ 7 & 0.86343 & -7.97997 \\ 8 & 0.82252 & 6.51420 \\ 9 & 0.82252 & -6.51420 \\ 10 & 0.84768 & 5.11226 \\ 11 & 0.84768 & -5.11226 \\ 12 & 0.89091 & 0.83008 \\ 13 & 0.89091 & -0.83008 \\ 14 & 0.85657 & 1.80876\end{array}$

$\begin{array}{llr}15 n & 0.85657 & -1.80676 \\ 16 & 0.81777 & 3.48803 \\ 17 & 0.81777 & -3.48803 \\ \text { COMMAN I : } & & \end{array}$

COMMAND NUMBER

NONPARAYETRIC ESTIMATION OF THE TRANSFER

FUNCTION AND IMPULSE RESPONSE

This command results in a nonparametric estimation (via FFTs and spectral smoothing techniques) of the transfer function between the input and output arrays. From the nonparametric frequency response, an inverse FFT results in an estimate of the impulse response.

This impulse response estimate is accurate oniy if 256 or fewer points of the input and output series were used.

USER CONTROL OVER THE COMAMAND

The user is not required to enter any input.

Certain details of the command are controlled by operation parameters. Any operation parameters may be changed by the command 9 .

The lag vindow operation parameter, LaG, controls the size of the lag window used in the smoothing of the spectrum. It may take on the values or 2 , corresponding to spectral estimate will result with a value of 2 . It has a default value of 1 .

The KSAMP operation parameter is the resampling factor. The user can control the upper frequency of the spectral plots with this parameter. It can take on the values 1, 2, 4, or 8 corresponding to the entire, half, rate. It has a default value of 8 .

$$
\text { Example of comerid? }
$$

EST. DF TRANSFER FUNCTION ANI IMFULSE RESFONSE

WARNING: SOME FOINTS OF THE ILFULSE RESFOHSE WILL RE ALIASEI,

GAIN

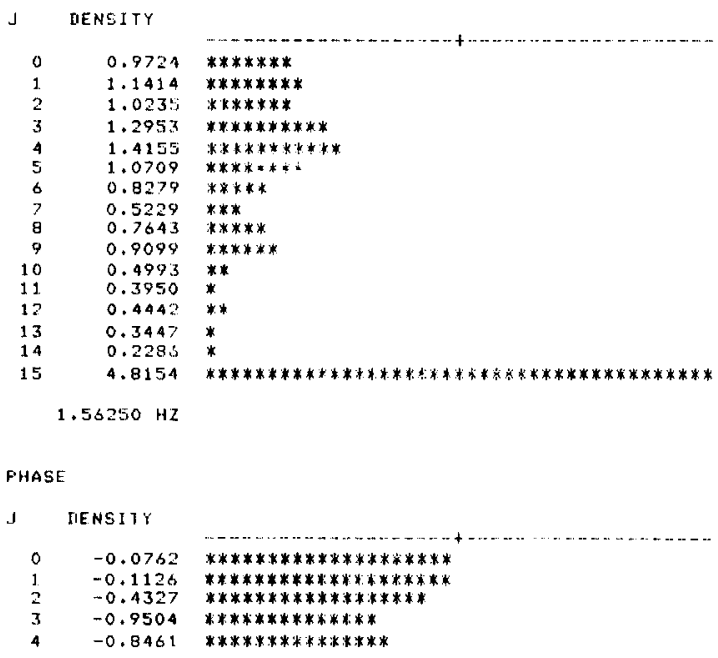




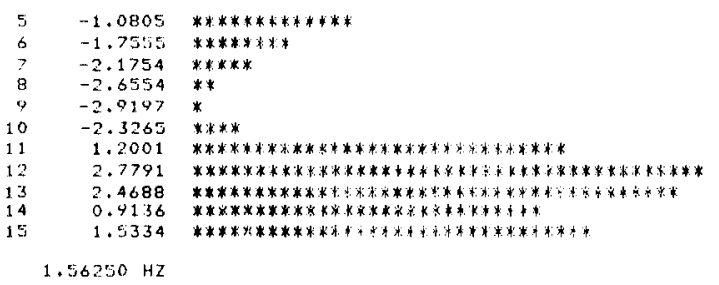

IMFULSE RESFONSE

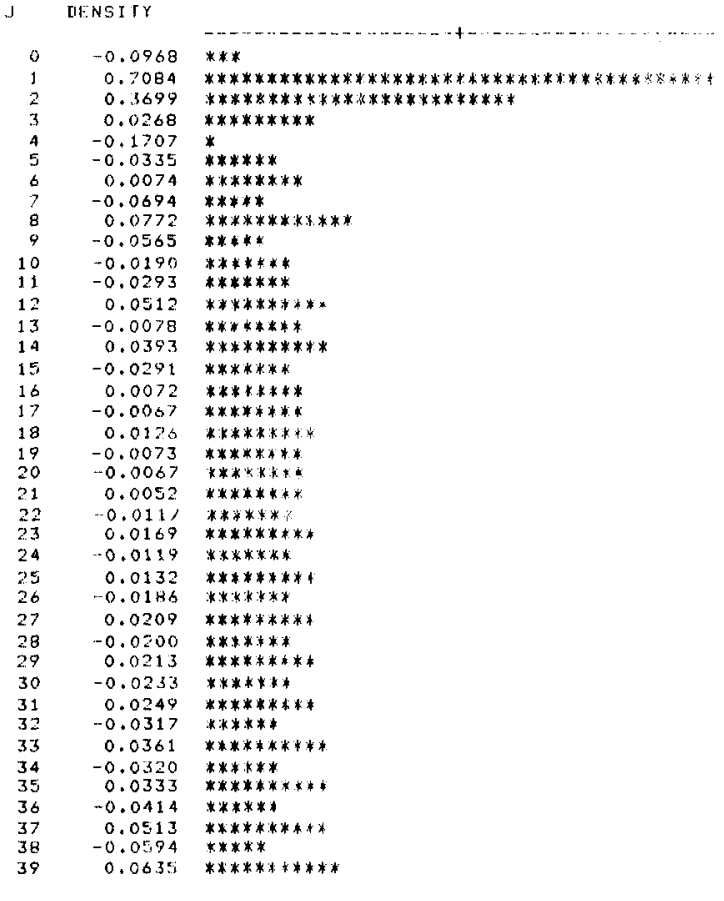

COMMANII:

COMGAND MUMBer B

\section{SPECIPICATION OF DIFTERENCE PARANTTERS AND THE}

CASE RAMG

\section{PUNCTION OP THE COMANAN}

This command results in the specification of of the case range and difference parameters for the input and output arrays. The case range can specify any starting and stopping point within the array size. Differencing parameters of out and output arrays.

COMMAND OUTPUT

If the detrending factor is set to detrend, then the slope, intercept, and variance of the possibly differenced data within the specified
USER CONTROL OVER THE COMMAND

Specification of the case range and the difference parameters must be done by the user interactively or from a command file.

The program types:

THE CURRENT CASE RANGE (IX IY)

If the endpoints $I X$ and $I Y$ are those desired, the user can type in $0 s$ in response and $I X$ and $I Y$ will not be changed. If not, the appropriate numbers should be maximum array size. Both must be greater than 0 .

For the difference parameters, the progran types:

THE CURRENT INPUT DIFFERENCE PARAMETER (IX)

THE CURRENT OUTPUT DIFFERENCE PARAMETER (IX)

Again, if the numbers in parentheses are those desired, a response causes no change. Otherwise, type a 0,1 , or 2 .

Certain details of the command are controlled by operation parameters. Any operation parameters may be changed by the command 9 .

The operation parameter, ITREND, controls whether the input and output arrays are detrended within the case range. If ITREND equals 1 , then detrending is done. The default value is 1 .

Example of comand $a$

THE CURFENT CASE RANGE( 1 512)23,512

THE CURRENT INFUT IIFFERENCE FAROMETER: 0$) 0$

THE CURRENT DUTFUT DIFFERENCE FAKAMETEKC O10

$\begin{array}{rlrc} & \text { SLOFE } & \text { INTERCEPT } & \text { SIGHAL VTFIINWCE } \\ \text { INFUT } & -0.00148 & 0.39598 & 4.58565 \\ \text { OUTFUT } & -0.00261 & 0.69719 & 5.13456\end{array}$

COMMANI:

COMOWND NUMBER 9

CHANGING OPERATION PARAMETERS

FUNCTION OF THE COMMAND

This command allows the user to change any of the 8 file.

The operation parameters are described below:

The NA operation parameter controls the number of normalized autocovariance lags plotted. It has a default value of 10 .

The NP operation parameter controls the number of partial autocorrelation lags plotted. It has a default value
of 10 .

The KSAMP operation parameter is the resampling factor. The user can control the upper frequency of the spectral plots with this parameter. It can take on the values $1,2,4$, or 8 corresponding to the entire, half, fourth, or eighth of the Nyquist frequency $(1 / 2$ the sampling rate. It has a default value of $B$.

The lag window operation parameter, LAG, controls the size of the lag window used in the smoothing of the spectrum. It may take on the values 1 or 2 , corresponding to a window length of $1 / 2$ or $1 / 4$ the array size. A smoother spectral estimate will result with a value of 2 . It has a
default value of 
The length of the estimated impulse response used to calculate the noise series is controlled by the operation parameter, IMP. It has a default value of 30 . It should be large enough so that significant values of the impulse response are not missed.

For the input univariate analysis:

The NPW parameter controls the number of partial autocorrelation lags plotted. It has a default value of 17. NPw is the number of autoregressive parameters with which the input is approximated.

The operation parameter, ITREND, controls whether the input and output arrays are detrended within the case range If ITREND equals 1 , then detrending is done. The default value is 1 .

The operation parameter, IDEV, controls the device from which the commands are read. If it equals 5 , the command which the commands are read. If it equals 5 , the command device is the

COMAND OUTPUT

The list of operation parameters and their allowable range are listed.

USER CONTROL OVER THE COMMAND

Each operation parameter is printed with the current value in parentheses. If the user desires to change the value, he types in the desired value. Typing a 0 results in no change to the parameter.

Example:

NUMBER OF AUTOCORRELATIONS (I $\mathbf{x}$ ) 0 IX

The number of autocorrelation lags printed will remain

Example of commang 9

9

LIST OF OFERATION FARAMETEFS
NO. AUTOCDRRELATION LAGS (HF TO 50 )

NO. FARTIAL AUYOCORFELATION LAGS CUF TO 50

LAG WINOIW FALTOR = $1 / 2$ TO THE FACTOEIL

RESAMFLING FACTOK $=1,2,4,8$

1 IS BEST FDF OBTAINING A MIISE ECT LUTIFE.

IMFULSE RESFONSE TENGIH FUR ESIIMATING NGISE (UIF 1050 )

NO. F'REWHI TENING FOLES (UF TO 50 )

IIETRENDING FACTUR = 1 FIUF LIEIRENIIIML, -1 FIIF HOT

COMMAND INFUT REUICF $\because \mathrm{E}$, COF TERMINAL, 3 FOF FILE

NUMBEF OF AUTOCORFELATIONS 10 )

NUMEEF OF FARTIAL AUTOCORFELATIONS $10: 0$

THE LAG WINIOWR 110

THE IMFULSE RESFONSE LENGTH 30,0

THE CURRENT RESAMF' ING FACTOR: 814

THE NUMBEF OF PFEWHITENING FOLES $1 \%$,O

THE IIETRENDING FACTOR: $1: 0$

THE IIETRENDING FACTOR $1: 0$

COMMANO:

COMAND ROMBER 10

STOPPING The AMALgSis

FUNCTION OF THE COMQUND:

This command stops analysis on the current data set. If other data sets have been included in the data 1 ist, then the new data are read in.

Example of command 10

10

******* CAMERA 2 ********

COMMAND: 10

TYFE 1 FOF NEW FILE, O TO STOF', - 1 FOR MOF'E

TO IIO IN CUREENT FILE

STOF' -- 